Chueng-Ryong Ji® $\cdot$ Ziyue Li • Bailing Ma •

Alfredo Takashi Suzuki

\title{
Interpolating Quantum Electrodynamics
}

Received: 7 January 2018 / Accepted: 3 May 2018 / Published online: 29 May 2018

(C) Springer-Verlag GmbH Austria, part of Springer Nature 2018

\begin{abstract}
We discuss the interpolation between the instant form dynamics (IFD) and the light-front dynamics (LFD) proposed by Dirac in 1949 and present its application to quantum electrodynamics (QED). Our analysis clarifies any conceivable confusion in the prevailing notion of the equivalence between the IFD at infinite momentum frame and the LFD. Entwining the fermion propagator interpolation with our previous works of the interpolating helicity spinors and the electromagnetic gauge field interpolation, we fasten the bolts and nuts necessary to launch the interpolating QED at least in the tree level.
\end{abstract}

\section{Introduction}

For the study of relativistic particle systems, Dirac [1] proposed three different forms of the relativistic Hamiltonian dynamics in 1949: i.e. the instant $\left(x^{0}=0\right)$, front $\left(x^{+}=\left(x^{0}+x^{3}\right) / \sqrt{2}=0\right)$, and point $\left(x_{\mu} x^{\mu}=a^{2}>0, x^{0}>0\right)$ forms. The instant form dynamics (IFD) of quantum field theories is based on the usual equal time $t=x^{0}$ quantization (units such that $c=1$ are taken here) and provides a traditional approach evolved from non-relativistic dynamics. The IFD makes a close contact with Euclidean space, developing temperature-dependent quantum field theory, lattice QCD, etc. The equal light-front time

This work was supported by the US DOE Contract No. DE-FG02-03ER41260 and in part by the FAPESP under process 2014/20892-2.

C.-R. Ji $(\varangle) \cdot$ B. Ma

Department of Physics, North Carolina State University, Box 8202, Raleigh, NC 27695-8202, USA

E-mail: crji@ncsu.edu

B. Ma

E-mail: bma4@ncsu.edu

Z. Li

First Leads, Inc., Durham, NC 27701, USA

E-mail: zli13@ncsu.edu

A. T. Suzuki

Instituto de Física Teórica-UNESP Universidade Estadual Paulista, Rua Doutor Bento Teobaldo Ferraz,

271 - Bloco II, Várzea da Barra Funda, São Paulo, São Paulo 01140-070, Brazil

Present address:

A. T. Suzuki

Department of Physics, La Sierra University, 4500 Riverwalk Pkwy, Riverside, CA 92505, USA

E-mail: asuzuki@lasierra.edu 
$\tau \equiv(t+z / c) / \sqrt{2}=x^{+}$quantization yields the front form dynamics, nowadays more commonly called lightfront dynamics (LFD) and provides an innovative approach to the study of relativistic dynamics. The LFD works strictly in Minkowski space, developing useful frameworks for the analyses of deep inelastic scattering (DIS), parton distribution functions (PDFs), deeply virtual Compton scattering (DVCS), generalized parton distributions (GPDs), etc. The quantization in the point form $\left(x^{\mu} x_{\mu}=a^{2}>0, x^{0}>0\right)$ is called radial quantization and this quantization procedure has been much used in string theory and conformal field theories [2] as well as in hadron physics [3-5]. Among these three forms of relativistic dynamics proposed by Dirac, the LFD carries the largest number (seven) of the kinematic (or interaction independent) generators leaving the least number (three) of the dynamics generators while both the IFD and the point form dynamics carry six kinematic and four dynamic generators within the total ten Poincaré generators. Indeed, the maximum number of kinematic generators allowed in any form of relativistic dynamics is seven and the LFD is the only one which possesses this maximum number of kinematic generators. Effectively, the LFD maximizes the capacity to describe hadrons by saving a lot of dynamical efforts in obtaining the QCD solutions that reflect the full Poincaré symmetries. In this presentation, we interpolate between the IFD and the LFD to link the LFD with the IFD which has been the traditional approach in relativistic dynamics and present its application to quantum electrodynamics (QED). Our works on interpolating the gauge fields and the fermion spinors have been presented in Ref. [6] and Ref. [7], respectively. In particular, we discussed the link between the Coulomb gauge in IFD and the light-front gauge in LFD [6] and the chiral representation of the helicity spinors interpolating between the IFD and the LFD [7]. Here, we entwine the fermion propagator interpolation with our previous works of the electromagnetic gauge field [6] and the helicity spinors [7] and fasten the bolts and nuts necessary to launch the interpolating QED at least in the tree level. Our analysis clarifies any conceivable confusion in the prevailing notion of the equivalence between the IFD at infinite momentum frame (IMF) [8] and the LFD.

In the next section, Sect. 2, we briefly review the gauge field and spinor interpolation between the IFD and the LFD discussing the prototype of QED scattering process " $e \mu \rightarrow e \mu$ ". In Sect. 3, we discuss the fermion propagator interpolation. Conclusion and outlook follow in Sect. 4.

\section{Gauge Field and Spinor Interpolation Between IFD and LFD}

The IFD and the LFD can be interpolated [9-11] by an interpolation angle between the ordinary time $t$ and the light-front time $\tau$. Introducing the interpolating scattering amplitude $[6,7,11]$ that links the corresponding time-ordered amplitudes between the two forms of dynamics, we discussed the physical meaning of the kinematic transformations in contrast to the dynamic transformations by means of checking the invariance of each individual time-ordered amplitude for an arbitrary interpolation angle. Using this interpolation method, we were able to also trace a dramatic change of the longitudinal boost's characteristics, namely, from "dynamic" in IFD to "kinematic" in LFD. This provides a distinct advantage to LFD as it can save a lot of dynamical effort in hadron phenomenology effectively and efficiently obtaining the QCD solutions that reflect the full Poincaré symmetries.

To trace the forms of relativistic quantum field theory between IFD and LFD, we take the following convention of the space-time coordinates to define the interpolation angle [6,7,9-11]:

$$
\left[\begin{array}{l}
x^{\hat{+}} \\
x^{\widehat{-}}
\end{array}\right]=\left[\begin{array}{cc}
\cos \delta & \sin \delta \\
\sin \delta & -\cos \delta
\end{array}\right]\left[\begin{array}{l}
x^{0} \\
x^{3}
\end{array}\right],
$$

in which the interpolation angle is allowed to run from 0 through $45^{\circ}, 0 \leq \delta \leq \frac{\pi}{4}$. All the indices with the wide-hat notation signify the variables with the interpolation angle $\delta$. For the limit $\delta \rightarrow 0$ we have $x^{\hat{+}}=x^{0}$ and $x^{-}=-x^{3}$ so that we recover usual space-time coordinates although the z-axis is inverted while for the other extreme limit, $\delta \rightarrow \frac{\pi}{4}$ we have $x^{\widehat{ \pm}}=\left(x^{0} \pm x^{3}\right) / \sqrt{2} \equiv x^{ \pm}$which leads to the standard light-front coordinates. Of course, the same interpolation applies to the momentum variables.

In Ref. [6], we developed the electromagnetic gauge field propagator interpolated between the IFD and the LFD and found that the light-front gauge $A^{+}=0$ in the LFD is naturally linked to the Coulomb gauge $\boldsymbol{\nabla} \cdot \mathbf{A}=0$ in IFD. We identified the dynamical degrees of freedom for the electromagnetic gauge fields as the transverse photon fields and clarified the equivalence between the contribution of the instantaneous interaction and the contribution from the longitudinal polarization of the virtual photon. Our results for the gauge propagator and time-ordered diagrams clarified whether one should choose the two-term form [12-14] or the three-term form [15-20] for the gauge propagator in LFD. Our transverse photon propagator in LFD 


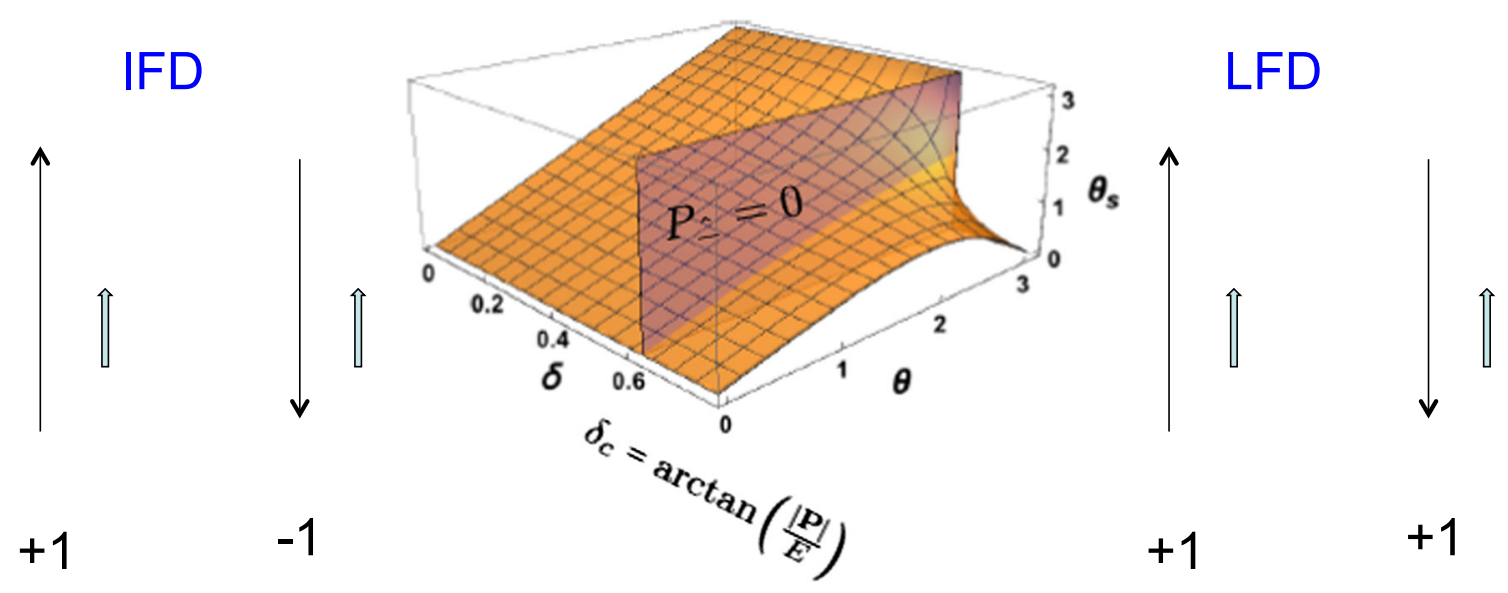

Fig. 1 The dependence of $\theta_{s}$ of a positive helicity spinor on the $\theta$ angle and the interpolation angle $\delta$ in the middle panel. We take a particle with mass $M=1 \mathrm{GeV}$ and total momentum $|\mathbf{P}|=1 \mathrm{GeV}$ as our illustrative example. The bifurcation point appears at the critical interpolation angle $\delta_{c}=\arctan \left(\frac{|\mathbf{P}|}{E}\right) \approx 0.61548$. The left and right panels depict the difference of the helicity definition in IFD and LFD, respectively. The helicity in IFD depends on the reference frame while the helicity in LFD is independent of the reference frame

assumes the three-term form, but the third term cancels the instantaneous interaction contribution. Thus, one can use the two-term form of the gauge propagator for effective calculation of amplitudes if one also omits the instantaneous interaction from the Hamiltonian. But if one wants to show equivalence to the covariant theory, all three terms should be kept because the instantaneous interaction is a natural result of the decomposition of Feynman diagrams, and the third term in the propagator is necessary for the total amplitudes to be covariant. We also see that the photon propagator was derived according to the generalized gauge that links the Coulomb gauge to light-front gauge and thus the three-term form appears appropriate in order to be consistent with the appropriate gauge.

In Ref. [7], we derived the generalized helicity spinor that links the instant form helicity spinor to the light-front helicity spinor. For a given generalized helicity spinor, the spin direction does not coincide with the momentum direction in general. Thus, we studied how the spin orientation angle $\theta_{s}$ changes in terms of both $\delta$ and the angle $\theta$ that defines the momentum direction of the particle. In Fig. 1, the dependence of $\theta_{s}$ of a positive helicity spinor on the $\theta$ angle is shown with respect to the interpolation angle $\delta$. In IFD $(\delta=0)$, the spin direction $\theta_{s}$ coincides with the momentum direction $\theta$ for the positive helicity as shown in the left panel as well as in the middle panel. However, the helicity in IFD depends on the reference frame. If the observer moves faster than the positive helicity spinor, then the direction of the momentum becomes opposite to the spin direction and the spinor of the helicity changes from +1 to -1 as depicted in the left panel of Fig. 1. In contrast, the helicity defined in LFD is independent of the reference frame as depicted in the right panel of Fig. 1. We have detailed the increment of the angle difference $\theta-\theta_{s}$ with the increment of the interpolation angle $\delta$ in Ref. [7], which bifurcates at a critical interpolation angle $\delta_{c}$. As illustrated in Fig. 1, we found $\delta_{c}=\arctan \left(\frac{|\mathbf{P}|}{E}\right)$. The IFD and the LFD separately belong to the two different branches bifurcated and divided out at the critical interpolation angle $\delta_{c}$. This bifurcation indicates the necessity of the distinction in the spin orientation between the IFD and the LFD and clarifies any conceivable confusion in the prevailing notion of the equivalence between the IMF formulated in IFD and the LFD.

Using the generalized helicity spinors, we computed all 16 helicity amplitudes and their squares (or probabilities) for the prototype of QED scattering process " $e \mu \rightarrow e \mu$ " [7]. This computation provided an explicit demonstration of the whole landscape picture for the helicity amplitudes and amplitude-squares that depend on the reference frame as well as the interpolation angle. To exhibit the boundaries appearing in the helicity amplitudes due to the the bifurcation of the branches distinguished by the critical interpolation angle $\delta_{c}$ as discussed above, we consider here almost back-to-back " $e \mu \rightarrow e \mu$ " scattering with $\theta=\pi-0.001$ essentially collinear in $z$ direction shown in Fig. 2 . As $\delta_{c}$ is obtained from the condition $P_{\simeq}=0$, all the initial and final particles that move essentially in the $\pm z$ direction yield the corresponding four boundaries upon the boost of the reference frame in the $\pm z$ direction as shown in Fig. 3. For an illustration, all of the colliding particles' helicities are taken as positive to yield the helicity amplitude $++\rightarrow++$ in Fig. 3 . The striking 


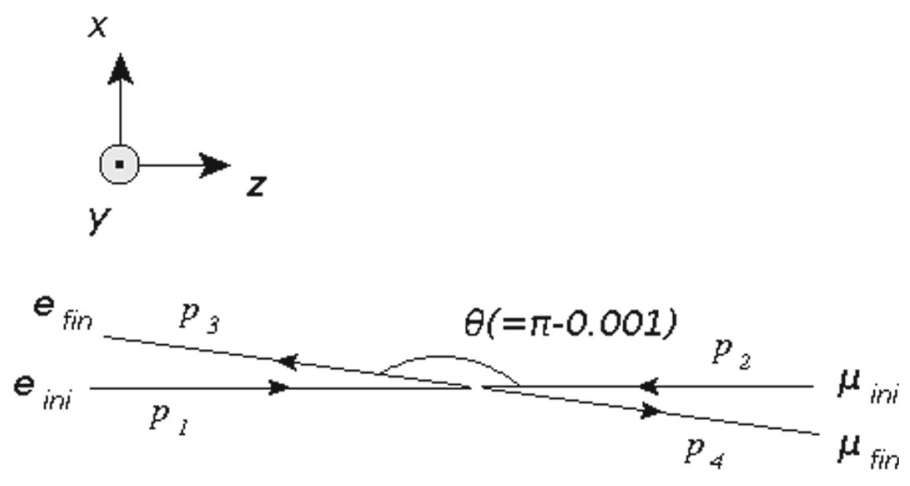

Fig. 2 Almost back-to-back collinear $e-\mu$ scattering

$$
++\rightarrow++(\theta=\pi-0.001)
$$

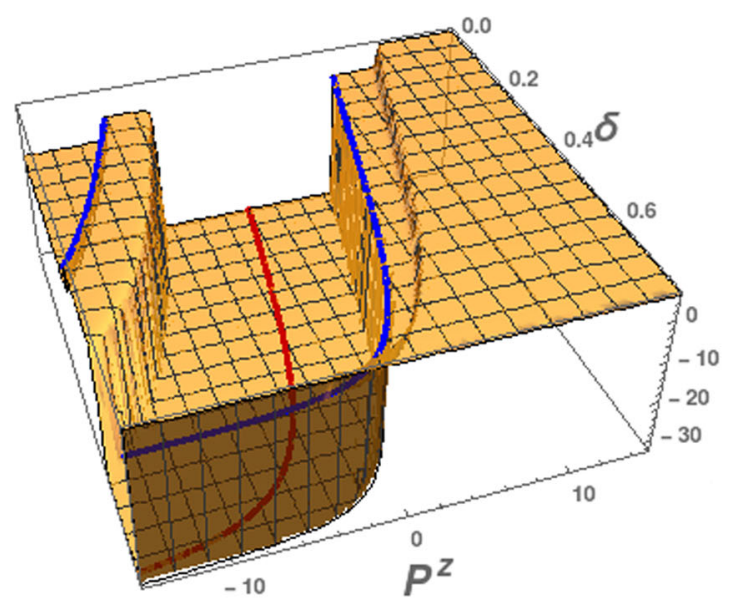

(a)

$$
++\rightarrow++(\theta=\pi-0.001)\left(m_{e}^{\prime}=0.7 m_{e}\right)
$$

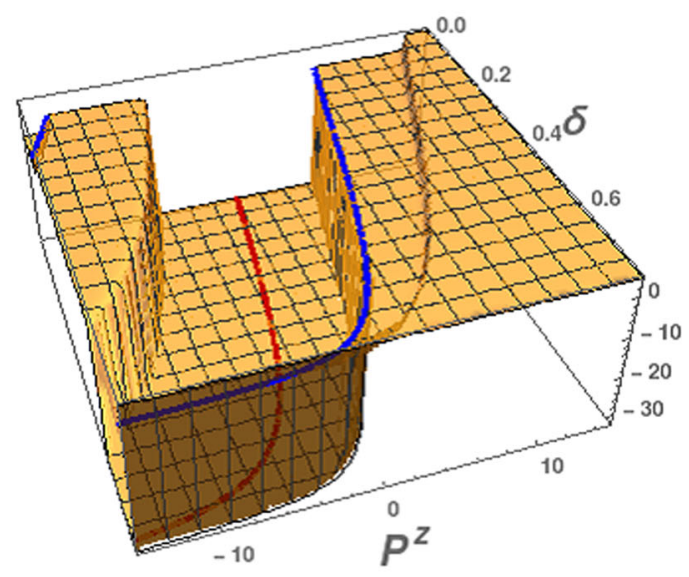

(b)

Fig. 3 Four boundaries appearing in almost back-to-back collinear $e-\mu$ scattering. The right panel depicts the movement of the boundaries for the smaller mass particles

feature of the interpolating helicity amplitudes is the appearance of the four boundaries in the IFD $(\delta=0)$ while one can see clearly that the helicity amplitudes in LFD $(\delta=\pi / 4)$ is completely independent of the reference frames or the total center of momentum (CM) $P^{z}$. The two blue lines indicate the two boundaries caused by the spin flip of each of the two incoming scattering particles while the red curve inside the two blue lines starts out in the $\mathrm{CM}$ frame in the $\delta=0$ limit and maintains the same amplitude value throughout the whole range of interpolation angle. In our previous works $[6,7,11]$, the J-shaped red curve was identified as universally given by $P^{z}=-\sqrt{\frac{s(1-\mathbb{C})}{2 \mathbb{C}}}$ where $\mathbb{C}=\cos 2 \delta$ and the Mandelstam variable $s$ is the total $\mathrm{CM}$ energy square. While the " $\mu$ " mass is taken same in both panels as $m_{2}=m_{4}=1.5 \mathrm{GeV} / \mathrm{c}$, the " $e$ " mass dependence of the boundaries is exhibited by the apparent movement of the two outside boundaries from $m_{1}=m_{3}=1 \mathrm{GeV} / \mathrm{c}$ in the left panel to $m_{1}=m_{3}=0.7 \mathrm{GeV} / \mathrm{c}$ in the right panel. As the " $e$ "mass $m_{1}$ and $m_{3}$ get smaller, the two outside boundaries move further away from the J-shaped red curve at the center. In the massless limit of " $e$ "mass $m_{1}=m_{3}=0 \mathrm{GeV} / \mathrm{c}$, the two outside boundaries disappear and only the two inside boundaries remain. 


\section{Interpolating Fermion Propagator}

Now that the spinor has been interpolated between IFD and LFD, the covariant Feynman propagator $\Sigma=\frac{q+m}{q^{2}-m^{2}}$ of the intermediate virtual fermion with the four-momentum $q$ and the mass $m$ can also be decomposed into the two interpolating time-ordered ones given by

$$
\Sigma_{a}=\frac{1}{2 Q^{\hat{+}}} \frac{\Phi_{a}+m}{q_{\hat{+}}-Q_{a \hat{+}}}, \quad \Sigma_{b}=\frac{1}{2 Q^{\hat{+}}} \frac{-Q_{b}+m}{-q_{\hat{+}}-Q_{b \hat{+}}},
$$

where

$$
\begin{aligned}
Q^{\hat{+}} & =\sqrt{q_{\hat{\imath}}^{2}+\mathbb{C}\left(\mathbf{q}_{\perp}^{2}+m^{2}\right)}, \\
Q_{a \hat{+}} & =\frac{-\mathbb{S} q_{a \hat{\imath}}+\sqrt{q_{a \hat{\imath}}^{2}+\mathbb{C} q_{a \perp}^{2}+\mathbb{C} m^{2}}}{\mathbb{C}}=\frac{-\mathbb{S} q_{a \hat{\varkappa}}+Q_{a}^{\hat{+}}}{\mathbb{C}}, \\
Q_{b \hat{+}} & =\frac{-\mathbb{S} q_{b \hat{-}}+Q_{b}^{\hat{+}}}{\mathbb{C}} .
\end{aligned}
$$

Here, $\mathbb{S}=\sin 2 \delta$ and the 4-momenta $q_{a}=q$ and $q_{b}=-q$ are those of the off-shell fermion and anti-fermion, while $Q_{a}$ and $Q_{b}$ are the corresponding on-shell 4-momenta with only their $\hat{+}$ components different from $q_{a}$ and $q_{b}$ 's, but given by Eqs. (4) and (5), respectively. In the light-front limit $\delta \rightarrow \frac{\pi}{4}$, i.e., $\mathbb{C} \rightarrow 0$, we get

$$
\Sigma_{a, \delta \rightarrow \frac{\pi}{4}}=\frac{q_{o n}+m}{q^{2}-m^{2}}, \quad \Sigma_{b, \delta \rightarrow \frac{\pi}{4}}=\frac{\gamma^{+}}{2 q^{+}},
$$

where $q_{o n}$ is a momentum 4-vector with its spacial part equal to that of $q\left(=p+k=p^{\prime}+k^{\prime}=q_{a}\right)$ but satisfies the Einstein energy-momentum relationship and $\Sigma_{b, \delta \rightarrow \frac{\pi}{4}}$ turns out to be the instantaneous contribution in the light-front propagator. This proves the usual light-front decomposition of the fermion propagator given by [21]

$$
\frac{1}{q-m}=\frac{\sum_{s} u(q, s) \bar{u}(q, s)}{q^{2}-m^{2}}+\frac{\gamma^{+}}{2 q^{+}}
$$

where the numerator $q_{o n}+m$ of $\Sigma_{a, \delta \rightarrow \frac{\pi}{4}}$ in Eq. (6) is replaced by the spin sum of the on-shell spinor product $\sum_{s} u(q, s) \bar{u}(q, s)$.

\section{Conclusion and Outlook}

Entwining the fermion propagator interpolation with our previous works of the interpolating helicity spinors and the electromagnetic gauge field interpolation, we have now completed all the necessary ingredients to establish the interpolating QED at least in the tree level. Any conceivable confusion between the IMF and the LFD has been clarified. Our complete work including the application to the Compton scattering and the two-photon production in $e^{+} e^{-}$annihilation is forthcoming to be presented elsewhere.

\section{References}

1. P.A.M. Dirac, Rev. Mod. Phys. 21, 392-399 (1949)

2. S. Fubini, A.J. Hanson, R. Jackiw, Phys. Rev. D 7, 1732 (1973)

3. LYa. Glozman, W. Plessas, K. Varga, R.F. Wagenbrunn, Phys. Rev. D 58, 094030 (1998)

4. R.F. Wagenbrunn, S. Boffi, W. Klink, W. Plessas, M. Radici, Phys. Lett. B 511, 33 (2001)

5. R.F.T. Melde, K. Berger, L. Canton, W. Plessas, R.F. Wagenbrunn, Phys. Rev. D 76, 074020 (2007)

6. C.-R. Ji, Z. Li, A.T. Suzuki, Phys. Rev. D 91, 065020 (2015)

7. Z. Li, M. An, C.-R. Ji, Phys. Rev. D 92, 105014 (2015)

8. S. Weinberg, Phys. Rev. 150, 1313 (1966)

9. K. Hornbostel, Phys. Rev. D 45, 3781-3801 (1992)

10. C.-R. Ji, C. Mitchell, Phys. Rev. D 64, 085013 (2001)

11. C.-R. Ji, A.T. Suzuki, Phys. Rev. D 87, 065015 (2013)

12. R. Perry, A. Harindranath, K. Wilson, Phys. Rev. Lett. 65, 2959 (1990) 
13. R. Perry, A. Harindranath, Phys. Rev. D 43, 4051 (1991)

14. D. Mustaki, S. Pinsky, J. Shigemitsu, K. Wilson, ibid 43, 3411 (1991)

15. G. Leibbrandt, Phys. Rev. D 29, 1699 (1984)

16. P. Srivastava, S. Brodsky, Phys. Rev. D 64, 045006 (2001)

17. A.T. Suzuki, J.H.O. Sales, Nucl. Phys. A 725, 139 (2003)

18. A.T. Suzuki, J.H.O. Sales, Mod. Phys. Lett. A 19, 1925 (2004)

19. A.T. Suzuki, J.H.O. Sales, Mod. Phys. Lett. A 19, 2831 (2004)

20. A. Misra, S. Warawdekar, Phys. Rev. D 71, 125011 (2005)

21. S.-J. Chang, T.-M. Yan, Phys. Rev. D 7, 1147 (1973) 